wild-type animals injected with FIV-Cre. Having demonstrated that expression of the IL-1 $1 \beta^{X A T}$ construct was sufficient to drive a sustained neuroinflammatory response within the mouse hippocampus, the researchers crossed this transgene into the APPSwe/PS1 1 E9 mouse model of AD. Surprisingly, 4 weeks after FIV-Cre injections were administered, plaque area fraction and frequency were, respectively, $59 \%$ lower and $46 \%$ lower in APPSwe/PS1 $\delta$ E9 mice exposed to sustained IL-1 $\beta$-driven neuroinflammation than in unexposed APPSwe-PS1 $\delta$ E9 mice. Hippocampal insoluble amyloids $\beta_{40}$ and $\beta_{42}$ were also reduced in the APPSwe-PS1 $\delta \mathrm{Eg}^{+}$ IL-1 $\beta$ XAT mice compared with APPSwe-PS1 $\delta$ E9 mice. Given the ability of microglia to phagocytose amyloid, the researchers surmise that the plaque amelioration seen in mice exposed to sustained IL-1 $\beta$ expression might be attributable to the enhanced microglial activation seen in these animals and could explain why antiinflammatory drugs have been unsuccessful in the treatment of $A D$.

Original article Shaftel SS et al. (2007) Sustained hippocampal IL-1 $\beta$ overexpression mediates chronic neuroinflammation and ameliorates Alzheimer plaque pathology. J Clin Invest 117: 1595-1604

\section{Resistance exercise slows functional decline in amyotrophic lateral sclerosis}

The utility of strengthening exercise for patients with amyotrophic lateral sclerosis (ALS) is controversial. In animal studies, high-intensity endurance exercise has been shown to be detrimental. Evidence suggests, however, that lowerintensity endurance exercise might be of benefit. Dal Bello-Haas et al. have investigated the effects of adding a thrice-weekly home-based resistance exercise program to a standard daily upper and lower extremity stretching program.

The study randomized 27 individuals with early-stage ALS to a program of stretching only $(n=14)$ or stretching plus resistance exercises $(n=13)$. Four individuals in the stretching-only group and five in the resistance group did not complete the 6-month follow-up period. None of the patients who dropped out did so because they believed the program was detrimental to their condition, but three withdrawals (1 stretching only; 2 with resistance exercise) were due to perceived lack of efficacy or to disease progression. At 6 months, the ALS Functional Rating Scale (ALSFRS) score was significantly better in the resistance-exercise group than in the stretching-only group $(P=0.02)$, as were ALSFRS upper and lower extremity subscale scores and physical function subscale scores on the SF-36 ${ }^{\circledR}$ quality of life questionnaire (Medical Outcomes Trust, Waltham, MA). No intervention-related adverse events were observed; resistance exercise had no negative effects on forced vital capacity, and seemed to slow decline in leg strength.

The authors recommend that patients in the early stages of ALS be encouraged to participate in nonfatiguing resistance exercise under the supervision of a qualified physical therapist; however, optimum programs are yet to be defined.

Original article Dal Bello-Haas V et al. (2007) A randomized controlled trial of resistance exercise in individuals with ALS. Neurology 68: 2003-2007

\section{Baseline brain function predicts response to restorative therapy for stroke}

Restorative interventions for stroke aim to improve outcome by increasing the function of surviving brain areas. In a recent Stroke paper, Cramer et al. report an inverse correlation between brain function at baseline and behavioral gains following restorative therapy. They conclude that measurement of brain function following stroke might assist clinicians in the selection of appropriate rehabilitation therapies.

In this prospective, unblinded study, 24 patients with chronic stroke were randomized to 6 weeks of rehabilitation therapy with or without cortical stimulation delivered by an implanted device. Degree of motor cortex activation (i.e. change in functional MRI signal between rest and motor task performance) was measured at study start and following therapy. Motor task performance was assessed by arm motor Fugl-Meyer (FM) score. After 6 weeks of therapy, 18 of the 24 patients showed improved motor task performance as indicated by arm motor FM score; however, no significant difference in change in arm motor FM score was noted between the stimulation and no-stimulation groups. Bivariate analyses found that stroke volume, a lower degree of activation in ipsilesional primary motor cortex at baseline, 